\title{
Modos de Ocupación del Bosque Patagónico de la Vertiente Oriental de Los Andes: Aportes desde la Ecología Isotópica
}

\author{
Human Occupation of the Eastern Patagonian Andean Forest: Insights from the Isotopic \\ Ecology
}

\author{
Pablo Marcelo Fernández ${ }^{\mathrm{i}}$ y Augusto Tessone $\mathrm{i}^{\mathrm{ii}}$
}

\begin{abstract}
RESUMEN
En este trabajo se discute el aporte de los isótopos estables del carbono y del nitrógeno al problema de los modos de ocupación humana del bosque de la vertiente oriental de los Andes. Se presenta una caracterización inicial de su ecología isotópica sobre la base de 58 valores de plantas y herbívoros y dos de restos óseos humanos, comprobándose que es posible discriminar señales isotópicas asociadas a recursos de bosque y evaluar el grado de permanencia y/o intensidad de empleo de este bioma en el pasado.
\end{abstract}

Palabras Clave: Poblamiento, Isótopos Estables, Bosque, Patagonia

\begin{abstract}
In this paper we discuss the contribution of stable isotopes of carbon and nitrogen to the problem of human occupation of the forest on the eastern slope of the Andes. An initial characterization of forest isotopic ecology is presented on the basis of 58 plants and herbivores values and two human values. It is possible to discriminate isotopic signals of forest resources and to assess the degree of permanence and / or intensity of use of forest in the past.
\end{abstract}

Key Words: Human Occupation, Stables Isotopes, Forest, Patagonia

\footnotetext{
i CONICET- Instituto Nacional de Antropología y Pensamiento Latinoamericano, 3 de Febrero 1370 (C1426BJN) Ciudad Autónoma de Buenos Aires, Argentina. Correo-e: pablomfernandez69@yahoo.com.ar

ii INGEIS-CONICET/UBA, Instituto de Geocronología Isotópica, Pabellón INGEIS - Ciudad Universitaria, Ciudad Autónoma de Buenos Aires, Argentina. Correo-e: atessone@ ingeis.uba.ar
}

Recibido: 28-01-2013 Revisado: 10-10-2013 Aceptado: 02-03-2014 


\section{INTRODUCCIÓN}

En la vertiente oriental de los Andes, el registro arqueológico en los bosques comprendidos entre $40^{\circ} 20^{\prime} \mathrm{S}$ y $43^{\circ} 10^{\prime} \mathrm{S}$ indica la existencia de diferentes modos de ocupación y/o explotación de este bioma a lo largo del tiempo y del espacio. Las ocupaciones previas a 3500 años AP son escasas, observándose un progresivo incremento en la cantidad de contextos con posterioridad a esta fecha (Fernández et al. 2013). Para este último lapso, la explotación del bosque ha sido pensada a partir de dos posturas generales, una en la que este bioma es aprovechado desde la estepa y otra que plantea que ciertas poblaciones vivían en el bosque de manera más o menos permanente. La primera se corresponde con los modelos propuestos para las áreas de Traful (Neuquén) y Los Alerces (Chubut) los que, con ligeras variaciones, proponen una ocupación logística del bosque a través de circuitos de movilidad que incluían la estepa y el ecotono bosqueestepa (Arrigoni 1997, Silveira 1999). La segunda postura fue propuesta para las áreas de Meliquina (Neuquén) y Nahuel Huapi (Neuquén - Río Negro) y en ambos casos involucra la presencia de poblaciones procedentes de la vertiente occidental de los Andes y el manejo de cultígenos (Lezcano et al. 2010, Pérez y Erra 20II). Estas posiciones pueden ser consideradas como parte de la gama de estrategias posibles de ser implementadas dentro del bosque andino patagónico, variabilidad que aún debe ser explorada. En ese sentido, la investigaciones que desarrollamos en el suroeste de Río Negro-noroeste de Chubut sugieren una tercera opción, la de poblaciones vinculadas con la estepa que habrían incrementado su permanencia en el bosque con posterioridad a 1700 años AP (Fernández et al. 20I3).

Entendemos que un modo novedoso de evaluar propuestas sobre el uso del bosque en Patagonia es desde la perspectiva de los isótopos estables del carbono y del nitrógeno. Si bien en la arqueología patagónica las paleodietas humanas han sido interpretadas en relación con la importancia relativa de los recursos de ambientes terrestres y marinos (Yesner et al. 1991, Barberena 2002, Favier Dubois et al. 2009, entre otros), trabajos recientes sugieren que los valores empobrecidos de ${ }^{15} \mathrm{~N}$ podrían constituir la marca distintiva del ambiente de bosque, inaugurando la posibilidad de ponderar el aporte de recursos animales de este bioma (Barberena et al. 20II, Méndez et al. 2014, Tessone et al. 20l4).
Con el propósito de explorar los alcances de esta vía de análisis presentamos los resultados de un programa de investigación orientado a la construcción de la ecología isotópica del bosque en la vertiente oriental de los Andes, que creemos permitirá evaluar los modos de uso del bosque, en especial los aspectos relacionados con el grado de permanencia $y / o$ intensidad de empleo de dicho ambiente. En ese sentido, se espera responder si la mayor regularidad en el uso del bosque, planteada sobre la base de otras líneas de evidencia, puede detectarse en una señal isotópica que refleje el consumo preferencial de recursos disponibles en este ambiente.

\section{MATERIALESY MÉTODOS}

La construcción de la ecología isotópica se basó en el análisis de plantas y herbívoros -modernos y arqueológicos- de distintos sectores de las provincias de Río Negro y Chubut. Las muestras fueron recuperadas en el ambiente de bosque o en el ecotono bosque-estepa y corresponden a: diez especies de árboles y arbustos; huemul (Hippocamelus bisulcus) y roedores del género Ctenomys y de la familia Caviidae. Las plantas fueron seleccionadas teniendo en cuenta la composición de la dieta de Hippocamelus bisulcus siguiendo a Galende et al. (2005). A modo comparativo se incorporan al análisis valores de plantas de la estepa de Santa Cruz (Tessone et al. 2014) y de guanacos de Río Negro y Chubut (Gómez Otero 2007, Favier Dubois et al. 2009, Tessone et al. 2014) junto a muestras de roedores del género Ctenomys del sitio Campo Moncada 2 (estepa, Chubut). Con el propósito de explorar la aplicación de los resultados obtenidos se analizaron dos muestras humanas arqueológicas recuperadas en los sitios Población Anticura (Río Negro) y Chacra de Lobos (Chubut).

El procesamiento de las muestras, tanto de vegetales como de restos óseos, se realizó en los laboratorios del Instituto de Geocronología y Geología Isotópica (INGEIS, CONICET-UBA) siguiendo procedimientos estándares (Tykot 2004). Las mediciones de las relaciones isotópicas se realizaron en este laboratorio (AIE) y en el de Isótopos Ambientales de la Universidad de Waterloo, Canadá (EILAB). El desvío estándar informado para los valores ${ }^{13} \mathrm{C}$ y ${ }^{15} \mathrm{~N}$ es de $\pm 0,3 \%$. En las muestras modernas 
se aplicó un factor de corrección para compensar el "efecto industrial" que genera un empobrecimiento de los valores de $\delta^{13} \mathrm{C}$ atmosféricos. La metodología utilizada es la propuesta por Long et al. (2005), la cual considera el año de muerte o recolección de la muestra para calcular la magnitud del factor de corrección. En relación con los huemules modernos ver detalles del cálculo en Tessone et al. (20I4).

\section{RESULTADOSY DISCUSIÓN}

Los valores isotópicos de $\delta^{13} \mathrm{C}$ y $\delta^{15} \mathrm{~N}$ obtenidos en el estudio de las plantas, animales y humanos, junto con los detalles de procedencia, tejido analizado y cronología, se presentan en la Tabla I. Las Tablas 2 y 3 consignan la estadística descriptiva para plantas y animales usando como fuente los valores de la Tabla I y datos publicados para plantas y guanacos de la estepa. Las tendencias observadas se grafican en la Figura I. Para las plantas $(\mathrm{N}=38)$ los valores de ${ }^{13} \mathrm{C}$ se disponen entre $-30,3 \%$ \% $-25,7 \%$ \% los de ${ }^{15} \mathrm{~N}$ entre $-4,9 \%$ y 7,1 \%。 (Tabla 2). Este último valor y otros dos se presentan como outliers dentro del rango del bosque (Figura la) y se superponen con la distribución informada para el ${ }^{15} \mathrm{~N}$ en plantas de la estepa recolectadas en la provincia de Santa Cruz (Figura la, Tabla 2). Desconocemos la razón que provoca una diferencia tan marcada (de casi 9 \%o) con la mediana obtenida para árboles y arbustos del bosque, sólo podemos mencionar que los valores altos de ${ }^{15} \mathrm{~N}$ corresponden a dos especies arbustivas, notro (Embothrium coccineum) y maitencillo (Maytenus sp.). Mas allá de los outliers del bosque (ver también la muestra de notro AIE27676, Tabla I) se observa una segregación marcada de las medidas de tendencia central entre las señales isotópicas de las plantas del bosque y de la estepa, con diferencias entre las medianas de $3,5 \%$ y $5, I \%$ en el ${ }^{13} \mathrm{C}$ y el ${ }^{15} \mathrm{~N}$, respectivamente (Tabla 2 ).
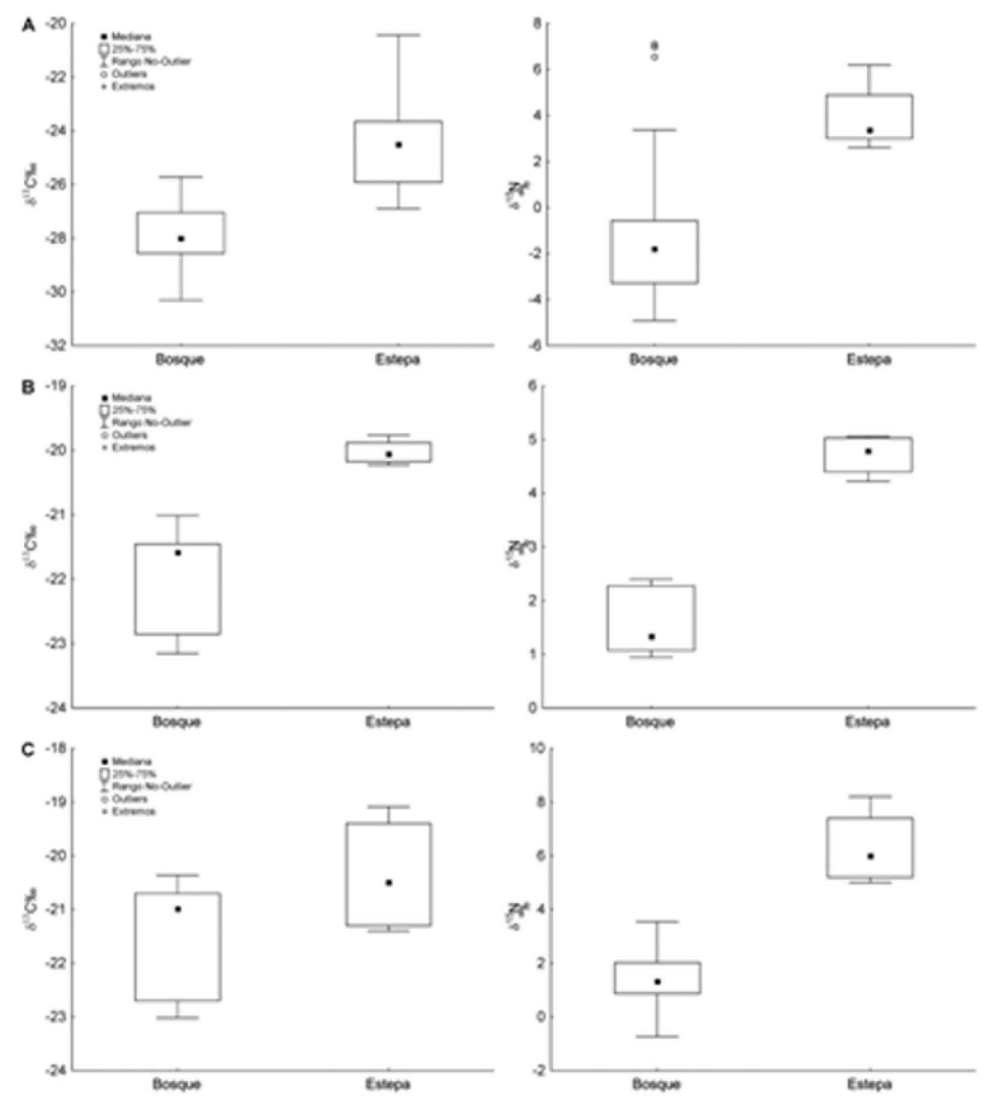

Figura 1: Gráficos de caja con la distribución de los valores isotópicos de plantas (A), roedores (B) y ungulados (C) por ambiente.

Figure 1: $\delta^{13} \mathrm{C}$ and $\delta^{15} \mathrm{~N}$ boxplot comparing plants, rodents, and ungulates from forest and steppe environments. 
Estos resultados sugieren que es posible discriminar, en la base de la cadena trófica, entre las señales isotópicas de las plantas que actualmente crecen en el bosque de aquellas que crecen en la estepa. Estas diferencias estarían relacionadas con el gradiente de precipitaciones que se da en sentido oeste-este en Patagonia (Austin y Sala 1999, Peri et al. 20I2).

La identificación de estas diferencias isotópicas en las plantas permite anticipar una segregación similar entre los herbívoros que consumen plantas en uno u otro ambiente. En el caso de los roedores, con especimenes recuperados de sitios arqueológicos del bosque y de la estepa, las diferencias isotópicas de las medianas son de I,5\% y $3,4 \%$ o para el ${ }^{13} \mathrm{C}$ y el ${ }^{15} \mathrm{~N}$, respectivamente (Figura IB, Tabla 3); no se observa superposición en los extremos de la distribución de ninguno de los isótopos y la diferencia es estadísticamente significativa (test Mann-Whitney para ambos isótopos $p=<0,05)$. Puede apreciarse una tendencia similar cuando se comparan los herbívoros de mayor tamaño que actualmente habitan estos ambientes en la Patagonia. En ambos isótopos las medidas de tendencia central de los huemules se encuentran empobrecidas respecto de los guanacos. En relación con el ${ }^{15} \mathrm{~N}$ la diferencia entre las medianas es de $4,6 \%$, estadísticamente significativa (test MannWhitney $p=<0,05$, Figura IC). Por el contrario, para el ${ }^{13} \mathrm{C}$ las medianas solo se distancian en $0,4 \%$ 。 $y$ no se registran diferencias estadísticas (test Mann-Whitney $p=>0,05$, Figura IC). No obstante, entre ambos herbívoros las diferencia es mayor sí consideramos las medias, I, 1\%。 (Tabla 3). Es posible que este resultado se deba a la escasa muestra de guanacos disponible para Río Negro y Chubut, las diferencias son mayores al comparar con la muestra más numerosa disponible para Santa Cruz (Tessone 2010).

\begin{tabular}{|c|c|c|c|c|c|c|c|c|}
\hline Especie / Taxón & $\begin{array}{l}\text { Localidad / } \\
\text { Sitio }\end{array}$ & Latitud / Longitud & $\begin{array}{l}\text { Código } \\
\text { laboratorio }\end{array}$ & Muestra & Cronología & ${ }^{13} \mathrm{C} \%$ & $\begin{array}{c}{ }^{13} \mathrm{C} \% \circ \\
\text { (Long et al. 2005) }\end{array}$ & $\delta^{15} \mathrm{~N} \%$ \\
\hline Embothrium coccineum & Lago Gutiérrez & $41^{\circ} 09^{\prime} \mathrm{S} 7 \mathrm{I}^{\circ} 24^{\prime} \mathrm{W}$ & EILAB2 I3597 & Hoja & Moderna & $-30,3$ & $-28,5$ & $-3,2$ \\
\hline Ribes magellanicum & Lago Gutiérrez & $41^{\circ} 09^{\prime} \mathrm{S} 7 \mathrm{I}^{\circ} 24^{\prime} \mathrm{W}$ & EILAB2 13598 & Hoja & Moderna & $-28,8$ & $-27,0$ & 1,9 \\
\hline Maytenus sp. & Lago Gutiérrez & $41^{\circ} 09^{\prime} \mathrm{S} 7 \mathrm{I}^{\circ} 24^{\prime} \mathrm{W}$ & EILAB213599 & Hoja & Moderna & $-29,8$ & $-27,9$ & 6.9 \\
\hline Nothofagus pumilio & Manso inferior & $41^{\circ} 37^{\prime} \mathrm{S} 7 \mathrm{I}^{\circ} 33^{\prime} \mathrm{W}$ & AIE27578 & Hoja & Moderna & $-29,1$ & $-27,2$ & $-2,7$ \\
\hline N. pumilio & Manso inferior & $41^{\circ} 37^{\prime} \mathrm{S} 7 \mathrm{I}^{\circ} 33^{\prime} \mathrm{W}$ & AIE27579 & Hoja & Moderna & $-30,1$ & $-28,3$ & $-1,6$ \\
\hline N. pumilio & Manso inferior & $41^{\circ} 37^{\prime} \mathrm{S} 7 I^{\circ} 33^{\prime} \mathrm{W}$ & AIE27580 & Hoja & Moderna & $-32,1$ & $-30,3$ & $-2,3$ \\
\hline N. pumilio & Manso inferior & $41^{\circ} 37^{\prime} \mathrm{S} 7 \mathrm{I}^{\circ} 33^{\prime} \mathrm{W}$ & AIE2758I & Hoja & Moderna & $-30,7$ & $-28,8$ & $-0,8$ \\
\hline N. dombeyi & Manso inferior & $41^{\circ} 37^{\prime S} 7 I^{\circ} 33^{\prime} \mathrm{W}$ & AIE27582 & Hoja & Moderna & $-30,6$ & $-28,7$ & $-2,9$ \\
\hline Austrocedrus chilensis & Manso inferior & $41^{\circ} 37^{\prime} \mathrm{S} 7 \mathrm{I}^{\circ} 33^{\prime} \mathrm{W}$ & AIE27659 & Hoja & Moderna & $-28,6$ & $-26,7$ & $-3,9$ \\
\hline A. chilensis & Manso inferior & $41^{\circ} 37^{\prime S} 7 I^{\circ} 33^{\prime} \mathrm{W}$ & AIE27660 & Hoja & Moderna & $-28,7$ & $-26,8$ & $-4,5$ \\
\hline A. chilensis & Manso inferior & $41^{\circ} 37^{\prime S} 7 I^{\circ} 33^{\prime} \mathrm{W}$ & AIE2766I & Hoja & Moderna & $-29,7$ & $-27,8$ & $-4,6$ \\
\hline A. chilensis & Manso inferior & $41^{\circ} 37^{\prime} \mathrm{S} 7 \mathrm{I}^{\circ} 33^{\prime} \mathrm{W}$ & AIE27662 & Hoja & Moderna & $-29,4$ & $-27,5$ & $-4,9$ \\
\hline A. chilensis & Manso inferior & $41^{\circ} 37^{\prime} \mathrm{S} 7 \mathrm{I}^{\circ} 33^{\prime} \mathrm{W}$ & AIE27663 & Hoja & Moderna & $-28,2$ & $-26,3$ & $-3,6$ \\
\hline N. pumilio & Manso inferior & $41^{\circ} 37^{\prime} \mathrm{S} 71^{\circ} 33^{\prime} \mathrm{W}$ & AIE27664 & Hoja & Moderna & $-29,8$ & $-27,9$ & $-3,6$ \\
\hline N. pumilio & Manso inferior & $41^{\circ} 37^{\prime} \mathrm{S} 7 \mathrm{I}^{\circ} 33^{\prime} \mathrm{W}$ & AIE27665 & Hoja & Moderna & $-29,2$ & $-27,3$ & $-3,8$ \\
\hline N. pumilio & Manso inferior & $41^{\circ} 37^{\prime} \mathrm{S} 7 \mathrm{I}^{\circ} 33^{\prime} \mathrm{W}$ & AIE27666 & Hoja & Moderna & $-30,1$ & $-28,2$ & $-0,6$ \\
\hline N. pumilio & Manso inferior & $41^{\circ} 37^{\prime} \mathrm{S} 7 \mathrm{I}^{\circ} 33^{\prime} \mathrm{W}$ & AIE27667 & Hoja & Moderna & $-30,3$ & $-28,4$ & $-0,1$ \\
\hline N. dombeyi & Manso inferior & $41^{\circ} 37^{\prime} \mathrm{S} 7 \mathrm{I}^{\circ} 33^{\prime} \mathrm{W}$ & AIE27668 & Hoja & Moderna & $-29,7$ & $-27,8$ & $-2,8$ \\
\hline N. dombeyi & Manso inferior & $41^{\circ} 37^{\prime} \mathrm{S} 7 I^{\circ} 33^{\prime} \mathrm{W}$ & AIE27669 & Hoja & Moderna & $-30,4$ & $-28,5$ & $-4,7$ \\
\hline N. antarctica & Manso inferior & $41^{\circ} 37^{\prime} \mathrm{S} 7 \mathrm{I}^{\circ} 33^{\prime} \mathrm{W}$ & AIE27670 & Hoja & Moderna & $-27,9$ & $-26,0$ & $-1,8$ \\
\hline N. antarctica & Manso inferior & $41^{\circ} 37^{\prime} \mathrm{S} 7 \mathrm{I}^{\circ} 33^{\prime} \mathrm{W}$ & AIE2767I & Hoja & Moderna & $-27,8$ & $-25,9$ & $-1,6$ \\
\hline N. antarctica & Manso inferior & $41^{\circ} 37^{\prime} \mathrm{S} 7 \mathrm{I}^{\circ} 33^{\prime} \mathrm{W}$ & AIE27672 & Hoja & Moderna & $-28,1$ & $-26,2$ & $-0,2$ \\
\hline N. antarctica & Manso inferior & $41^{\circ} 37^{\prime} \mathrm{S} 7 \mathrm{I}^{\circ} 33^{\prime} \mathrm{W}$ & AIE27673 & Hoja & Moderna & $-30,4$ & $-28,5$ & 0,0 \\
\hline
\end{tabular}


Modos de Ocupación del Bosque Patagónico de la Vertiente Oriental de Los Andes:Aportes desde la Ecología Isotópica

\begin{tabular}{|c|c|c|c|c|c|c|c|c|}
\hline N. pumilio & Manso inferior & $41^{\circ} 37^{\prime} \mathrm{S} 71^{\circ} 33^{\prime} \mathrm{W}$ & AIE27674 & Hoja & Moderna & $-29,4$ & $-27,5$ & $-0,5$ \\
\hline E. coccineum & Manso inferior & $41^{\circ} 37^{\prime} \mathrm{S} 7 \mathrm{I}^{\circ} 33^{\prime} \mathrm{W}$ & AIE27675 & Hoja & Moderna & $-30,9$ & $-29,0$ & 7,1 \\
\hline E. coccineum & Manso inferior & $41^{\circ} 37^{\prime} \mathrm{S} 71^{\circ} 33^{\prime} \mathrm{W}$ & AIE27676 & Hoja & Moderna & $-30,2$ & $-28,3$ & 3,3 \\
\hline E. coccineum & Manso inferior & $41^{\circ} 37^{\prime} \mathrm{S} 71^{\circ} 33^{\prime} \mathrm{W}$ & AIE27677 & Hoja & Moderna & $-31,5$ & $-29,6$ & 6,5 \\
\hline $\begin{array}{l}\text { Maytenus boaria } \\
\text { magellanicus }\end{array}$ & Manso inferior & $41^{\circ} 37^{\prime} \mathrm{S} 7 \mathrm{I}^{\circ} 33^{\prime} \mathrm{W}$ & AIE27678 & Hoja & Moderna & $-27,9$ & $-26,0$ & $-1,5$ \\
\hline M. boaria magellanicus & Manso inferior & $41^{\circ} 37^{\prime} \mathrm{S} 71^{\circ} 33^{\prime} \mathrm{W}$ & AIE27679 & Hoja & Moderna & $-27,6$ & $-25,7$ & $-1,8$ \\
\hline M. boaria magellanicus & Manso inferior & $41^{\circ} 37^{\prime} \mathrm{S} 7 \mathrm{I}^{\circ} 33^{\prime} \mathrm{W}$ & AIE27680 & Hoja & Moderna & $-28,2$ & $-26,3$ & $-1,4$ \\
\hline M. boaria magellanicus & Manso inferior & $41^{\circ} 37^{\prime} \mathrm{S} 71^{\circ} 33^{\prime} \mathrm{W}$ & AIE2768I & Hoja & Moderna & $-29,0$ & $-27,1$ & $-1,5$ \\
\hline Gaultheria sp. & Manso inferior & $41^{\circ} 37^{\prime} \mathrm{S} 71^{\circ} 33^{\prime} \mathrm{W}$ & AIE27682 & Hoja & Moderna & $-30,4$ & $-28,5$ & 2,1 \\
\hline Gaultheria sp. & Manso inferior & $41^{\circ} 37^{\prime} \mathrm{S} 71^{\circ} 33^{\prime} \mathrm{W}$ & AIE27683 & Hoja & Moderna & $-29,8$ & $-27,9$ & 1,2 \\
\hline Maytenus chubutensis & Manso inferior & $41^{\circ} 37^{\prime} \mathrm{S} 71^{\circ} 33^{\prime} \mathrm{W}$ & AIE28840 & Hoja & Moderna & $-30,5$ & $-28,6$ & $-3,2$ \\
\hline M. chubutensis & Manso inferior & $41^{\circ} 37^{\prime} \mathrm{S} 71^{\circ} 33^{\prime} \mathrm{W}$ & AIE2884I & Hoja & Moderna & $-30,9$ & $-29,1$ & $-3,3$ \\
\hline Diostea juncea & Manso inferior & $41^{\circ} 37^{\prime} \mathrm{S} 71^{\circ} 33^{\prime} \mathrm{W}$ & AIE28842 & Hoja & Moderna & $-30,2$ & $-28,3$ & $-1,6$ \\
\hline D. juncea & Manso inferior & $41^{\circ} 37^{\prime} \mathrm{S} 71^{\circ} 33^{\prime} \mathrm{W}$ & AIE28843 & Hoja & Moderna & $-29,9$ & $-28,0$ & $-1,8$ \\
\hline D. juncea & Manso inferior & $41^{\circ} 37^{\prime} \mathrm{S} 71^{\circ} 33^{\prime} \mathrm{W}$ & AIE28844 & Hoja & Moderna & $-30,4$ & $-28,5$ & $-2,2$ \\
\hline Caviidae & Manso inferior & $41^{\circ} 35^{\prime} \mathrm{S} 71^{\circ} 31^{\prime} \mathrm{W}$ & AIE28526 & Mandibula & $480 \mathrm{AP}$ & $-22,0$ & - & 1,3 \\
\hline Caviidae & Manso inferior & $41^{\circ} 35^{\prime} \mathrm{S} 71^{\circ} 31^{\prime} \mathrm{W}$ & AIE28828 & Mandíbula & $480 \mathrm{AP}$ & $-22,8$ & - & 2,4 \\
\hline Caviidae & Manso inferior & $41^{\circ} 35^{\prime} \mathrm{S} 71^{\circ} 31^{\prime} \mathrm{W}$ & AlE28837 & Mandibula & $480 \mathrm{AP}$ & $-23,1$ & - & 2,0 \\
\hline Caviidae & Manso inferior & $41^{\circ} 35^{\prime} \mathrm{S} 71^{\circ} 31^{\prime} \mathrm{W}$ & AIE28836 & Mandíbula & $480 \mathrm{AP}$ & $-21,0$ & _ & 1,0 \\
\hline Ctenomys sp. & Manso inferior & $41^{\circ} 35^{\prime} \mathrm{S} 71^{\circ} 31^{\prime} \mathrm{W}$ & AIE28839 & Mandíbula & $<280$ AP & $-21,5$ & - & 2,2 \\
\hline Ctenomys sp. & Manso inferior & $41^{\circ} 35^{\prime} \mathrm{S} 71^{\circ} 31^{\prime} \mathrm{W}$ & AIE28832 & Cráneo & $280-300 \mathrm{AP}$ & $-21,4$ & - & 0,9 \\
\hline Ctenomys sp. & Manso inferior & $41^{\circ} 35^{\prime} \mathrm{S} 71^{\circ} 31^{\prime} \mathrm{W}$ & AlE2883I & Mandíbula & $480 \mathrm{AP}$ & $-21,4$ & - & 1,0 \\
\hline Hippocamelus bisulcus & Manso inferior & $41^{\circ} 35^{\prime} \mathrm{S} 71^{\circ} 31^{\prime} \mathrm{W}$ & AIE28528 & Cúbito & $480 \mathrm{AP}$ & $-20,3$ & - & 2,8 \\
\hline H. bisulcus & Manso inferior & $41^{\circ} 35^{\prime} \mathrm{S} 71^{\circ} 32^{\prime} \mathrm{W}$ & AIE28530 & Costilla & $<1500 \mathrm{AP}$ & $-20,4$ & _- & 3,5 \\
\hline H. bisulcus & Cholila & $42^{\circ} 30^{\prime} \mathrm{S} 71^{\circ} 31^{\prime} \mathrm{W}$ & AIE28527 & Metatarso & $<1890$ AP & $-23,0$ & - & 1,9 \\
\hline H. bisulcus & Los Alerces & $42^{\circ} 54^{\prime} \mathrm{S} 71^{\circ} 35^{\prime} \mathrm{W}$ & AlE28529 & Cúbito & I460 AP & $-20,9$ & - & 0,3 \\
\hline H. bisulcus * & Ñirihuau & $41^{\circ} 20^{\prime} \mathrm{S} 71^{\circ} 21^{\prime} \mathrm{W}$ & EILAB I9596I & Falange & Moderno & $-22,4$ & $-21,2$ & 0,8 \\
\hline H. bisulcus * & Ñirihuau & $41^{\circ} 20^{\prime} \mathrm{S} 71^{\circ} 21^{\prime} \mathrm{W}$ & EILAB 195963 & Vértebra & Moderno & $-21,5$ & $-20,7$ & 1,3 \\
\hline H. bisulcus * & $\begin{array}{l}\text { Lago } \\
\text { Futalaufquen }\end{array}$ & $42^{\circ} 50^{\prime} \mathrm{S} 71^{\circ} 42^{\prime} \mathrm{W}$ & EILAB 195960 & Vértebra & Moderno & $-22,0$ & $-20,8$ & 1,2 \\
\hline H. bisulcus * & Los Alerces & $42^{\circ} 50^{\prime} \mathrm{S} 71^{\circ} 42^{\prime} \mathrm{W}$ & EILAB 195958 & Vértebra & Moderno & $-23,7$ & $-22,8$ & 2,0 \\
\hline H. bisulcus * & Lago La Plata & $44^{\circ} 52^{\prime} \mathrm{S} 71^{\circ} 50^{\prime} \mathrm{W}$ & EILAB213596 & Vértebra & Moderno & $-23,9$ & $-22,7$ & $-0,7$ \\
\hline Ctenomys sp. & Piedra Parada & $42^{\circ} 38^{\prime} \mathrm{S} 70^{\circ} 06^{\prime} \mathrm{W}$ & AIE28834 & Mandíbula & $780 \mathrm{AP}$ & $-19,7$ & - & 4,5 \\
\hline Ctenomys sp. & Piedra Parada & $42^{\circ} 38^{\prime} \mathrm{S} 70^{\circ} 06^{\prime} \mathrm{W}$ & AlE28833 & Mandibula & $780 \mathrm{AP}$ & $-19,9$ & - & 4,2 \\
\hline Ctenomys sp. & Piedra Parada & $42^{\circ} 38^{\prime} \mathrm{S} 70^{\circ} 06^{\prime} \mathrm{W}$ & AlE28829 & Mandíbula & $780 \mathrm{AP}$ & $-20,1$ & - & 5,0 \\
\hline Ctenomys sp. & Piedra Parada & $42^{\circ} 38^{\prime} \mathrm{S} 70^{\circ} 06^{\prime} \mathrm{W}$ & AlE28838 & Mandíbula & $860 \mathrm{AP}$ & $-20,2$ & - & 5,0 \\
\hline Homo sapiens & $\begin{array}{l}\text { Población } \\
\text { Anticura }\end{array}$ & $41^{\circ} 35^{\prime} \mathrm{S} 71^{\circ} 31^{\prime} \mathrm{W}$ & EILAB2 I 3594 & Pelvis & I550 AP & $-20,7$ & - & 6,9 \\
\hline H. sapiens & $\begin{array}{l}\text { Chacra de } \\
\text { Lobos }\end{array}$ & $42^{\circ} 01^{\prime} \mathrm{S} 71^{\circ} 33^{\prime} \mathrm{W}$ & AA69914 & Cráneo & $698 \mathrm{AP}$ & $-22,1$ & - & 7,6 \\
\hline
\end{tabular}

Tabla 1: Valores isotópicos de plantas, animales y humanos de Río Negro y Chubut. ${ }^{*}$ Valor publicado en Tessone et al. (2014).

Table 1: $\delta^{13} C$ and $\delta^{15} \mathrm{~N}$ dataset of plants, animals and humans from Río Negro and Chubut. ${ }^{*}$ Data published in Tessone et al. (2014). 
Estos resultados sugieren que los isótopos del carbono, pero muy especialmente del nitrógeno, pueden usarse como marcadores geográficos para discriminar entre estos dos ambientes terrestres de Patagonia. A la vez, permiten explorar en qué medida los recursos de la estepa y del bosque contribuyeron a la dieta de los cazadoresrecolectores patagónicos, lo que también funciona como un indicador de la intensidad y/o frecuencia de uso de estos ambientes. Utilizando un fraccionamiento medio de $1 \% \circ$ para $d^{13} \mathrm{C}$ y de 4\%。 para el $d^{15} \mathrm{~N}$ (Bocherens y Drucker 2003) la dieta estimada de los individuos recuperados en los sitios Población Anticura es de $d^{13} \mathrm{C}-21,7 \%$ y $\mathrm{d}^{15} \mathrm{~N}$ $2,9 \%$ y de $d^{13} \mathrm{C}-23,1 \%$ y $d^{15} \mathrm{~N} 3,6 \%$ o para Chacra de Lobos, lo que sugiere el consumo de recursos alimenticios con señales isotópicas del bosque. La antigüedad de estas muestras (Tabla I) coincide con el planteo que en los últimos 1700 años AP se habría dado un mayor grado de permanencia y/o intensidad de empleo del bosque. Aun cuando las tendencias registradas en la ecología isotópica $y$ en las muestras humanas deben ser reforzadas con el análisis de más muestras, consideramos que lo expuesto confirma la utilidad de los estudios isotópicos para conocer los modos de uso del bosque patagónico en el pasado. Análisis en curso de muestras de otras latitudes permitirán discutir el alcance de nuestra propuesta en bosques con condiciones ecológicas distintas.

\begin{tabular}{|l|c|c|c|c|}
\cline { 2 - 5 } \multicolumn{1}{c|}{} & \multicolumn{2}{c|}{ Bosque } & \multicolumn{2}{c|}{ Estepa } \\
\cline { 2 - 5 } \multicolumn{1}{c|}{} & $\mathrm{d}^{13} \mathrm{C} \%$ & $\mathrm{~d}^{15} \mathrm{~N} \% \circ$ & $\mathrm{d}^{13} \mathrm{C} \%$ & $\mathrm{~d}^{15} \mathrm{~N} \%$ \\
\hline $\mathrm{N}$ & 38 & 38 & 7 & 7 \\
\hline Media & $-27,8$ & $-\mathrm{I}, 2$ & $-24,3$ & 3,8 \\
\hline Mediana & $-27,9$ & $-\mathrm{I}, 8$ & $-24,4$ & 3,3 \\
\hline Desvío Estándar & $\mathrm{I}, 0$ & 3,0 & 2,0 & $\mathrm{I}, 2$ \\
\hline Mínimo & $-30,3$ & $-4,9$ & $-26,9$ & 2,6 \\
\hline Máximo & $-25,7$ & $7, \mathrm{I}$ & $-20,4$ & 6,1 \\
\hline
\end{tabular}

Tabla 2: Estadística descriptiva de los valores isotópicos de plantas por ambiente.

Table 2: Central tendency and dispersion of isotopic values of plant from forest and steppe environments.

\begin{tabular}{|c|c|c|c|c|c|c|c|c|}
\hline & \multicolumn{4}{|c|}{ Bosque } & \multicolumn{4}{|c|}{ Estepa } \\
\hline & \multicolumn{2}{|c|}{ Huemul } & \multicolumn{2}{|c|}{ Roedores } & \multicolumn{2}{|c|}{ Guanaco* } & \multicolumn{2}{|c|}{ Roedores } \\
\hline & $d^{13} \mathrm{C} \%$ & $d^{15} \mathrm{~N} \%$ & $d^{13} \mathrm{C} \%$ & $d^{15} \mathrm{~N} \%$ & $d^{13} \mathrm{C} \%$ & $d^{15} \mathrm{~N} \%$ & $d^{13} \mathrm{C} \%$ & $d^{15} \mathrm{~N} \%$ \\
\hline $\mathrm{N}$ & 9 & 9 & 7 & 7 & 5 & 5 & 4 & 4 \\
\hline Media & $-21,4$ & $\mathrm{I}, 4$ & $-21,9$ & $\mathrm{I}, 5$ & $-20,3$ & 6,3 & $-20,0$ & 4,7 \\
\hline Mediana & $-20,9$ & $\mathrm{I}, 3$ & $-21,5$ & $\mathrm{I}, 3$ & $-20,5$ & 5,9 & $-20,0$ & 4,7 \\
\hline Desvío Estándar & $\mathrm{I}, 0$ & $\mathrm{I}, 2$ & 0,7 & 0,6 & 1,0 & 1,3 & 0,2 & 0,3 \\
\hline Mínimo & $-23,0$ & $-0,7$ & $-23,1$ & 0,9 & $-21,4$ & 5,0 & $-20,2$ & 4,2 \\
\hline Máximo & $-20,3$ & 3,5 & $-21,0$ & 2,4 & $-19,1$ & 8,2 & $-19,7$ & 5,0 \\
\hline
\end{tabular}

Tabla 3. Estadística descriptiva de los valores isotópicos de muestras de herbívoros recuperados en el bosque y la estepa. Referencia: ${ }^{*}$ Gómez Otero (2007), Favier Dubois et al. (2009), Tessone et al. (2014).

Table 3: Central tendency and dispersion of isotopic values of herbivores from forest and steppe environments. References: ${ }^{*}$ Gómez Otero (2007), Favier Dubois et al. (2009), Tessone et al. (2014).

Agradecimientos: A M. M. Bianchi y C. Lavecchia por la recolección de las plantas utilizadas en el análisis. A la Administración de Parques Nacionales, en especial a Eduardo Ramilo y Soledad Caracotche, por sus estímulos y apoyo en el estudio del huemul. Las investigaciones fueron financiadas con los subsidios PIP CONICET 232/10 y UBACYT 09I y recursos financieros del INGEIS (CONICETUBA). 


\section{BIBLIOGRAFÍA}

Arrigoni, G. I. 1997. "Pintando entre lagos y bosques (las pinturas rupestres del Parque Nacional Los Alerces. Chubut). Actas y Memorias del XI Congreso Nacional de Arqueología Argentina (Cuarta Parte)". Revista del Museo de Historia Natural de San Rafael (Mendoza). Tomo XVI (I/4): 24I-268.

Austin, A. T. y O. E. Sala 1999. "Foliar ${ }^{15} \mathrm{~N}$ is negatively correlated with rainfall along the IGBP transect in Australia". Australian Journal Plant Physiologist 26: 293-295.

Barberena, R. 2002. Los Límites del mar: Isótopos Estables en Patagonia Meridional. Sociedad Argentina de Antropología, Colección Tesis de Licenciatura, Buenos Aires.

Barberena, R., C. Méndez, F. Mena y O. Reyes 20II. "Endangered species, archaeology, and stable isotopes: huemul (Hippocamelus bisulcus) isotopic ecology in centralwestern Patagonia (South America)". Journal of Archaeological Science 38: 2313-2323.

Bocherens, H. y D. G. Drucker 2003. "Trophic Level Isotopic Enrichment of Carbon and Nitrogen in Bone Collagen: Case Studies from Recent and Ancient Terrestrial Ecosystems". International Journal of Osteoarchaeology 13: 46-53.

Favier Dubois, C. M., F. Borella y R. H. Tykot 2009. "Explorando tendencias en el uso humano del espacio y los recursos en el litoral rionegrino (Argentina) durante el Holoceno medio y tardío". En Arqueología de Patagonia: una mirada desde el último confin, editado por M. Salemme, F. Santiago, M. Álvarez, E. Piana, M. Vázquez y E. Mansur, Tomo 2, pp. 985-997. Editorial Utopías, Ushuaia.

Fernández, P. M., M. Carballido Calatayud, C. Bellelli y M. Podestá 20 I3."Tiempo de cazadores. Cronología de las ocupaciones humanas en el valle del río Manso inferior (Río Negro)". En Tendencias teórico-metodológicas y casos de estudio en la arqueología de Patagonia. Editado por A. F. Zangrando, R. Barberena, A. Gil, G. Neme, M. Giardina, L. Luna, C. Otaola, S. Paulides, L. Salgán y A.Tivoli, pp. 167-175. Museo de Historia Natural de San Rafael, Sociedad Argentina de Antropología y Instituto Nacional de Antropología y Pensamiento Latinoamericano, Buenos Aires.

Galende, G., E. Ramilo y A. Beati 2005. "Diet of Huemul deer (Hippocamelus bisulcus) in Nahuel Huapi National Park, Argentina". Studies on Neotropical Fauna and Environment 40: I-5.

Gómez Otero, J. 2007. "Isótopos estables, dieta y uso del espacio en la costa atlántica centro septentrional y el valle inferior del río Chubut (Patagonia argentina)". En Arqueología de Fuego-Patagonia. Levantando piedras, desenterrando huesos... y develando arcanos, editado por F. Morello, M. Martinic, A. Prieto y G. Bahamondes, pp. 151-161. Universidad de Magallanes, Punta Arenas.
Lezcano, M. J., A. Hajduk y A. M. Albornoz 2010. "El menú a la carta en el bosque ientrada o plato fuerte?: una perspectiva comparada desde la zooarqueología del sitio El Trébol (lago Nahuel Huapi, Pcia. de Río Negro)". En Zooarqueología a principios del siglo XXI: aportes teóricos, metodológicos y casos de estudio, editado por M.A. Gutiérrez, M. De Nigris, P. M. Fernández, M. Giardina, A. F. Gil, A. Izeta, G. Neme y H. D. Yacobaccio, pp. 243257. Ediciones del Espinillo, Buenos Aires.

Long, E. S., R. A. Sweitzer, D. R. Diefenbach y M. Ben-David 2005. "Controlling for anthropogenically induced atmospheric variation in stable carbon isotope studies". Oecologia I46: I48-I56.

Méndez, C., R. Barberena, O. Reyes y A. Nuevo Delaunay 2014. "Isotopic Ecology and Human Diets in the Forest-Steppe Ecotone,Aisén Region, Central-Western Patagonia, Chile". International Journal of Osteoarchaeology 24: I87-20I (DOI: 10.1002/oa.2337).

Pérez, A. y G. Erra 20Il. "Identificación de maíz en vasijas recuperadas de la Patagonia Noroccidental Argentina". Magallania 39(2): 309-316.

Peri, P. L., B. Ladd, D. A. Pepper, S. P. Bonser, S. W. Lafan y W. Amelung 2012."Carbon $\left(\delta^{13} \mathrm{C}\right)$ and nitrogen $\left(\delta^{15} \mathrm{~N}\right)$ stable isotope composition in plant and soil in Southern Patagonia's native forests". Global Change Biology I8(I): 3II-32I.

Silveira, M. 1999. "Alero Cicuta (Departamento Los Lagos, Provincia del Neuquén,Argentina)". En Soplando en elViento. Actas de las Terceras Jornadas de Arqueología de la Patagonia, Pp. 56I-576. INAPL-Universidad Nacional del Comahue, NeuquénBuenos Aires.

Tessone, A. 2010. Arqueología y Ecología Isotópica. Estudio de isótopos estables de restos humanos del Holoceno tardío en Patagonia meridional. Tesis Doctoral Inédita, Facultad de Filosofía y Letras, Universidad de Buenos Aires.

Tessone, A., P. M. Fernández, C. Bellelli y H. Panarello 2014. " $\delta^{13} \mathrm{C}$ and $\delta^{15} \mathrm{~N}$ characterization of modern huemul (Hippocamelus bisulcus) from the Patagonian Andean forest. Scope and limitations of their use as a geographical marker". International Journal of Osteoarchaeology 24: 219-230.

Tykot, R. 2004."Stable Isotopes and diet:you are what you eat. En Physics Methods in Archaeometry", editado por M. Martini, M. Milazzo y M. Piacentini, Proceedings of the International School of Physics "Enrico Fermi" Course 154:433-444. Società Italiana di Fisica, Bologna, Italia.

Yesner D., M. J. Figuerero, R. Guichon y L. A. Borrero 1991. "Análisis de isótopos estables en esqueletos humanos: confirmación de patrones de subsistencia etnográficos para Tierra del Fuego". Shincal 3: 182-191. 